\title{
INVESTIGACIONES
}

\section{Enseñando matemáticas a futuros profesores en un sistema complejo: Formadores de profesores en universidades de Chile*}

\author{
Teaching mathematics in a complex system: Teacher educators in universities in Chile
}

\author{
Darinka Radovic ${ }^{a}$, Bernardita Peñafiel ${ }^{b}$,Ana N. SanMartín ${ }^{c}$, \\ Sofía Bustos ${ }^{d}$, Salomé Martinez \\ ${ }^{a}$ Centro de Modelamiento Matemático, UMI 2807 CNRS-Universidad de Chile. \\ Correo electrónico: dradovic@dim.uchile.cl \\ ${ }^{\text {b }}$ Centro de Modelamiento Matemático, UMI 2807 CNRS-Universidad de Chile. \\ Correo electrónico: bernarditapf@gmail.com

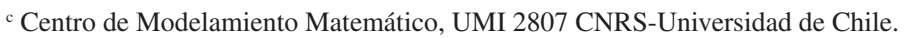 \\ Correo electrónico: anaansm@gmail.com \\ ${ }^{\mathrm{d}}$ Centro de Modelamiento Matemático, UMI 2807 CNRS-Universidad de Chile. \\ Correo electrónico: sbustos@dim.uchile.cl \\ e Departmento de Ingeniería Matemática, Centro de Modelamiento Matemático, \\ UMI 2807 CNRS-Universidad de Chile, \\ Correo electrónico: samartin@dim.uchile.cl
}

\begin{abstract}
RESUMEN
Aún cuando el rol de formadores de profesores es central en la generación de mejoras en la formación inicial docente, ha existido poca atención destinada a este actor tanto en investigación internacional como en Latinoamérica y Chile. Este paper reporta los resultados de un estudio cualitativo que explora experiencias de formadores de profesores de matemáticas en el ejercicio de roles e identidades correspondientes en diferentes contextos universitarios en Chile. En particular, explora cómo formadores de 18 universidades describen sus roles en estos contextos, y cómo la experiencia de acceso a recursos y apoyo impacta sus identidades profesionales. El artículo concluye sugiriendo que proveer apoyo y libertad para el ejercicio de múltiples roles puede tener un impacto positivo en el acercamiento de los formadores a su labor y a situaciones de cambio, y que incluso formadores con larga experiencia se pueden sentir inexperimentados cuando no perciben apoyos institucionales.
\end{abstract}

Palabras clave: identidad docente, Formación Inicial Docente, formadores de profesores, políticas públicas

\begin{abstract}
Although teacher educators are central in improving initial teacher education, there has been little attention into this actor both in international and Latin American research. This paper reports an exploratory qualitative study on the experiences of teacher educators enacting their roles and corresponding identities in different university contexts in Chile. It explores particularly how teacher educators from 18 universities describe their roles in these settings and how the experience of different access to resources and support impact their professional identities. It suggests that providing with support for implementing multiple roles can have a positive impact in teacher educators approach to their roles and change and that even experienced teachers can feel lacking experience when they are unsupported.

Keywords: teacher identity, initial teacher education, teacher educators, public policy
\end{abstract}

\footnotetext{
Este trabajo fue financiado a través de los Proyectos FONDECYT 3180291, CONICYT + PIA/ Concurso apoyo a centros científicos y tecnológicos de excelencia con financiamiento basal AFB170001 y FONDEF \#ID16I10119.
} 
Estudios Pedagógicos XLIV, $\mathrm{N}^{\circ}$ 3: 101-116, 2018

ENSEÑANDO MATEMÁTICAS A FUTUROS PROFESORES EN UN SISTEMA COMPLEJO: FORMADORES DE PROFESORES EN UNIVERSIDADES DE CHILE

\section{INTRODUCCIÓN}

La formación inicial de profesores es central en el logro de mejoras en el sistema educativo en general. Es en este espacio donde es posible promover nuevas visiones sobre la enseñanza y el aprendizaje (Putnam \& Borko, 2000) y donde se puede proveer a los futuros profesores con conocimientos y habilidades necesarias para educar a sus estudiantes para cumplir las demandas del siglo 21 (OECD, 2005). Considerando la gran cantidad de investigación que existe sobre profesores y estudiantes de pedagogía, es sorprendente la poca atención que se ha puesto en los formadores de profesores. Varios autores han sugerido que es necesario aumentar la investigación respecto de este actor, tanto en Latinoamérica y Chile (Cisternas, 2011; Gonzalez-Vallejos, 2018) como a nivel internacional (e.g. Lunenberg, Korthagen \& Swennen, 2007; Loughran, 2011), existiendo literatura emergente reciente en formación inicial.

La escasa literatura que se ha enfocado en los formadores de profesores ha descrito su trabajo como una profesión muy poco definida, incluso "esquizofrénica" (Lunenberg, Dengerink \& Korthagen, 2014). Diferentes estudios han resaltado que los formadores deben satisfacer múltiples roles, incluyendo enseñar, investigar, entrenar a estudiantes en práctica, desarrollar o modificar currículum, coordinar el trabajo con establecimientos educacionales, etc. (Celik, 2011; Lunenberg et al., 2014; Shagrir, 2015). Las principales dificultades que se han descrito en la literatura son las tensiones que existen entre estos roles y las correspondientes diferentes identidades que los formadores deben encarnar. Por ejemplo, investigación ha documentado los problemas que existen en la transición que los formadores deben hacer desde la enseñanza a niños en colegios a la enseñanza de adultos en la universidad (Dinkelman, Margolis \& Sikkenga, 2006; Swennen, Shagrir \& Cooper, 2009; Zeichner, 2005). En esta transición se ha reportado la existencia de tensiones que los formadores experimentan entre sus previas identidades y el desarrollo de sus nuevas identidades profesionales (Murray \& Kosnik, 2011; Williams \& Ritter 2010), incluidos nuevos roles como aquellos relacionados con la investigación (Guilfoyle, 1995). Estas diferentes "identidades" (por ejemplo, investigador-profesor) se ha visto que requieren de distintas habilidades y competencias (Koster, Brekelmans, Korthagen \& Wubbels, 2005) habilidades que incluso a veces se encuentran en conflicto (Murray \& Male, 2005; Contreras, Monereo \& Badia, 2010; Contreras, 2014).

La mayoría de la literatura centrada en las identidades de los formadores de profesores han utilizado o metodologías de autoreflexión, explorando en detalle las complejidades del tránsito de un contexto particular (usualmente la escuela) a otro (usualmente la universidad) (ej. Dinkelman et al., 2006; Swennen et al., 2009; Zeichner, 2005) o se ha centrado en concepciones individuales del rol y de las competencias necesarias sin explorar cómo estos pueden diferir en distintos contextos (Smith, 2005). La ausencia de influencias contextuales en la investigación sobre formadores de profesores es sorprendente, sobre todo considerando cuán variadas son las situaciones en las que estos educadores deben actuar (Gonzalez-Vallejos, 2018; William \& Berry, 2016). Considerando estos antecedentes, este estudio explorará distintas identidades que los formadores deben representar de forma de cumplir sus roles en universidades con demandas de política pública similares, pero en contextos muy diferentes. En particular, explorará las demandas y recursos disponibles para los formadores y cómo ellos usan estos recursos y demandas en la negociación de sus identidades de formadores. 


\section{IDENTIDADES DE PROFESORES Y DE FORMADORES DE PROFESORES: RELEVANCIA DE CONTEXTOS Y TRANSICIONES}

De manera de explorar cómo los formadores de profesores enfrentan distintas demandas, cuentan con distintos recursos y deben cumplir distintos roles, este estudio utiliza el concepto de Identidad desde una perspectiva social (por ejemplo, Holland, Lachicotte, Skinner \& Cain, 1998) y en particular una visión social de las identidades de los profesores que las entiende como múltiples, discontinuas y asociadas a contextos sociales específicos (Akkerman \& Meijer, 2011; Beijaard, Meijer \& Verloop, 2004; Olsen, 2008). Estos tres aspectos implican que las identidades de los profesores están constantemente en procesos de interpretación y re-interpretación, en lo que puede ser entendido como un proceso de desarrollo dirigido hacia quién me quiero convertir (Beijaard et al., 2004) y están asociadas a contextos específicos determinados por culturas locales de instituciones, pero también con culturas específicas de las disciplinas de enseñanza (Beauchamp \& Thomas, 2009). Siguiendo esto, las identidades de los profesores ya no pueden ser entendidas como unitarias y continuas, sino que son múltiples y diferentes según si son representadas en, por ejemplo, la sala de clases, el laboratorio de investigación, la reunión de dirección, la comisión de construcción de curriculum, etc.

Siguiendo la importancia de los contextos locales, muchos autores han explorado la influencia de estos en las posibilidades de desarrollo profesional de profesores en formación y en servicio. Estos estudios han confirmado que las prácticas locales de escuelas influyen fuertemente en el proceso de construcción de identidades profesionales en la transición desde la formación inicial a la práctica profesional (Legard Larson \& Kalmbach Phillips, 2005; Smagorinsky, Cook, Moore, Jackson \& Fry, 2004) o en los procesos de cambio de profesores en servicio para implementación de reformas o luego de participación en programas de formación profesional (Battey \& Franke, 2008; Gresalfi \& Cobb, 2011). Además, otros autores han demostrado que no existen sólo influencias de contextos locales sino también influencias más macro institucionales como la constante re-restructuración de la educación, la profesión docente y el currículum (Sachs, 2005). Por ejemplo, en Chile, Gellert, Espinoza \& Barbé (2013) describieron cómo una profesora que inicialmente había incorporado cambios de un programa de desarrollo docente, en el largo plazo cambió nuevamente sus prácticas debido a que el establecimiento educacional donde trabajaba estaba promoviendo un nuevo programa de educación. En este caso los autores hipotetizan que las rápidas reformas implementadas en este país dificultan que los profesores incorporen nuevas metodologías que permitan, por ejemplo, cambios en su identidad de profesores.

Específicamente en formadores de profesores la investigación ha sido menos intensa y numerosa que la investigación en profesores en formación y en servicio, pero evidencia grandes complejidades en la labor de formar profesores. Por ejemplo, Koster y colegas (2005) realizaron un perfil profesional de los formadores de profesores, mostrando que estos deben realizar múltiples diferentes tareas (por ejemplo, trabajar en su desarrollo profesional, desarrollar los programas de educación, revisando materiales, impartiendo cursos, evaluando, supervisando prácticas, desarrollo de políticas del sistema a través de por ejemplo el desarrollo del currículum, y realización de investigación), tareas que requerirían diferentes competencias (por ejemplo, competencias respecto al contenido, a la capacidad de comunicar reflexiones, de organizar, etc.). La importancia que los formadores 
Estudios Pedagógicos XLIV, $\mathrm{N}^{\circ}$ 3: 101-116, 2018

ENSEÑANDO MATEMÁTICAS A FUTUROS PROFESORES EN UN SISTEMA COMPLEJO: FORMADORES DE PROFESORES EN UNIVERSIDADES DE CHILE

le atribuían a estas distintas tareas y competencias variaba fuertemente en distintos tipos de instituciones, principalmente la importancia que se le atribuía a la investigación en universidades en comparación con instituciones de enseñanza vocacional y técnica.

Similares diferencias contextuales fueron encontradas por Murray \& Male (2005) en un estudio en el que exploraron las experiencias de transición de 28 formadores en sus entradas a siete diferentes programas de formación de profesores. En este caso los autores exploraron las distintas experiencias de estos formadores nóveles y encontraron que los formadores experimentaban mayores presiones a tomar nuevas identidades como investigadores en instituciones tradicionales/antiguas, mientras que los formadores que se integraron en instituciones nuevas no sentían esta presión.

Dengerink, Lunenberg y Kools (2015) por su parte exploraron las distintas necesidades de aprendizaje que experimentan formadores en instituciones de educación superior y formadores que realizan su labor en instituciones escolares (realizando predominantemente trabajo de tutorías a estudiantes en sus prácticas profesionales). Estos autores mostraron que en general los formadores de profesores en instituciones universitarias mostraban más interés en desarrollar sus habilidades de enseñanza y formadores en escuelas solicitaban mayor formación para realizar tareas como tutorías y seguimiento de prácticas.

En Chile, aún existe muy poca investigación centrada específicamente en formadores de profesores y esta naciente investigación ha mostrado múltiples complejidades que ellos y ellas deben enfrentar en el ejercicio de su labor. Por ejemplo, Montenegro y Medina (2014) en un análisis en profundidad del discurso de siete formadores respecto de su enseñanza, encontraron que distintas "ideologías" están en tensión y coexisten en el discurso de los formadores. Tanto la disciplina, la forma de enseñar, la práctica en aula escolar y la figura del estudiante y su futuro como profesor se constituyen como actores fundamentales respecto de cómo se toman decisiones pedagógicas y por lo tanto influyen finalmente en la práctica. Contreras y colegas $(2010 ; 2014)$ encontraron que formadores de distintas universidades reconocían distintas identidades, no siempre explícitamente relacionadas e incluso muchas veces en clara tensión. En las entrevistas además estos formadores identificaron numerosos incidentes críticos que influyen en su trabajo relacionados con características de las instituciones donde trabajaban, como la existencia de múltiples contradicciones y demandas institucionales (por ejemplo, bajas oportunidades de formación y colaboración) y las bajas oportunidades de encuentro y trabajo con otros (Contreras, 2014)

Uno de los problemas del ejercicio de múltiples roles e identidades asociadas se relaciona con la valoración que estos tienen dentro de las instituciones y en el contexto nacional. Por ejemplo, las tareas de enseñanza y acompañamiento de alumnos en práctica suelen imponer una gran carga de trabajo en los formadores, lo que implica bajas oportunidades para investigación y para influir en el currículum. Esto se ha relacionado con un menor estatus dentro de las instituciones, acceso a menores recursos y menor influencia en los programas y en sus políticas a largo plazo (Simmons \& Thompson, 2007). Sin embargo, cómo estos roles son distribuidos y valorados dentro de las instituciones dependerá fuertemente de los contextos locales, características de las instituciones e incluso contextos geográficos más macro donde las instituciones están insertas. Aprovechando el acceso a formadores que trabajan en contextos muy diversos dentro de Chile, el presente estudio busca explorar cómo estos contextos influyen el ejercicio y construcción del rol y en las identidades que formadores de profesores deben encarnar en instituciones de educación superior, específicamente en el área de las matemáticas. 


\section{CONTEXTO CHILENO}

Siguiendo numerosos reportes que han llamado la atención respecto de problemas de calidad en la formación inicial de profesores en Chile (ej. Comisión sobre Formación Inicial Docente, 2005; OECD, 2011), en los últimos 20 años el Ministerio de Educación ha promovido diversos programas y reformas orientados a promover mayor calidad, encontrándose en un estado de constante reforma (Ávalos \& de los Ríos, 2012). Entre los programas y reformas más importantes se incluyen la incorporación de criterios de acreditación más exigentes para las carreras y demandas más altas para la selección de los estudiantes, la promoción de entrada de alumnos de más alto rendimiento académico a través de la generación de becas, la generación de estándares de formación inicial y el desarrollo de pruebas que permitan evaluar el cumplimiento de estos estándares, entre otros. Además, se ha promovido el desarrollo de programas de mejoramiento a nivel institucional a través de la apertura de oportunidades de financiamiento estatal (Mecesup, 2016).

Todas las iniciativas descritas han estado insertas en un contexto de oferta educativa altamente diverso. En Chile la historia de reformas orientadas a mejoras en la formación inicial docente ha resultado en una alta diversificación de la oferta (Ávalos, 2014; Sotomayor \& Gysling, 2011). La de-profesionalización de la carrera de profesores que ocurrió durante la dictadura militar, seguida por la activa promoción de la privatización de la educación en los primeros gobiernos post-dictadura significó grandes cambios en la oferta educativa (Ávalos, 2002). Un aumento en los niveles de control de las universidades más tradicionales y la creciente demanda de matrícula generada por un explosivo aumento de la oferta privada de educación, generó un gran aumento de la oferta de programas con muy bajos requerimientos de entrada para los alumnos (Cox, Meckes \& Bascopé, 2010). Este aumento en la oferta produjo también una descentralización de la oferta educativa, existiendo nuevas universidades regionales que contrastan con instituciones de más larga data (públicas y tradicionales). Así se introduce la satisfacción de la demanda de educación universitaria en pequeñas ciudades introduciendo "sedes", las que usualmente cuentan con menor matrícula (Brunner, 2005). Como consecuencia, hoy en día existe en Chile importantes brechas entre instituciones, incluyendo grandes diferencias entre universidades públicas y privadas, tradicionales y no tradicionales, entre universidades con distintos requisitos de entrada e incluso distinta oferta para distintos mercados educativos. Por ejemplo, particularmente en matemáticas, el estudio TEDS-M mostró que las Universidades más tradicionales (públicas y privadas) ofrecen programas más completos y sus estudiantes alcanzan mejores resultados en pruebas de conocimientos disciplinarios y pedagógicos que universidades privadas no tradicionales (Ávalos \& Matus, 2010).

En respuesta a las nuevas demandas impuestas en el sistema a las universidades y a las nuevas definiciones de estándares desarrolladas en el país, muchos programas de formación docente han impulsado cambios en sus currículums. Cambios sustantivos ya habían sido promovidos por programas como el PFFID (Programa de Fortalecimiento de la Formación Inicial de Docentes) y por informes generados por la OCDE, incidiendo en la incorporación de prácticas progresivas y en la generación de mayor cantidad de cursos disciplinares específicos (Mineduc, 2005). Por ejemplo, en matemáticas dos estudios realizados cerca del año 2010 mostraron que la mayoría de los programas de educación básica de formación de profesores de matemáticas contaban con muy baja oferta de cursos específicos de esta disciplina y de la didáctica de la disciplina (Ávalos \& Matus, 2010; Varas et al., 2008), 
y muy baja cobertura de los contenidos de las bases curriculares de la enseñanza escolar (Ávalos \& Matus, 2010). En un estudio encargado por el Ministerio de Educación, para analizar características de los formadores de profesores y de los programas de cursos del área de matemática ofrecidos encontró que esta oferta había cambiado sustantivamente, encontrándose mayor cantidad de cursos disciplinarios y mayor cobertura del currículum escolar (Mineduc, 2016). Esto implica que gran parte de los programas de formación de profesores han sufrido importantes reformas curriculares y de planes, reformas que aún en algunas universidades siguen en curso.

Por último, los programas de educación de profesores en Chile exigen que sus formadores tengan preparación y experiencia en sala, pero pocos han incluido como requisito el grado de doctor, existiendo a su vez muchos formadores de profesores trabajando en jornadas parciales (72\%) (Ávalos \& Matus, 2010). Esto puede también influir en las potenciales demandas existentes en la satisfacción de roles de investigación al interior de las instituciones y en las condiciones generales de trabajo.

\section{METODOLOGÍA}

Durante el año 2017, una universidad tradicional y pública de Santiago de Chile implementó un programa de desarrollo profesional sobre enseñanza de las matemáticas para formadores de profesores de educación básica al que asistieron formadores de profesores de distintas regiones y universidades del país. Este estudio está basado en una serie de recogidas de información realizadas con estos formadores orientadas a entender las diferentes necesidades y contextos en las que estos formadores forman en el área de las matemáticas a futuros profesores básicos. La información fue recogida utilizando encuestas de caracterización inicial, grupos focales y entrevistas individuales.

\section{PARTICIPANTES}

En la encuesta de caracterización y en los grupos focales participaron 25 formadores de profesores y luego seis de ellos fueron entrevistados en mayor profundidad en una entrevista individual dos meses después de la primera recogida de datos. Del total, 23 de los formadores contaban con formación de posgrado: cuatro con diplomas de profundización, 13 con magister y tres con doctorado. Todos ellos se encontraban trabajando en 18 universidades diferentes, de un total de 45 instituciones que ofrecían formación de profesores de Educación Básica. Estas universidades están ubicadas en distintas zonas del país (cinco del norte, nueve del centro y de la RM y cuatro del sur), tienen distintos niveles de selectividad de sus estudiantes (seis no tienen mecanismos de selección; seis, nivel bajo; cuatro, mediano; y dos, alta selección) y de diferentes tamaños (13 pequeñas, cuatro medianas y una grande).

\section{PROCEDIMIENTOS DE RECOLECCIÓN DE INFORMACIÓN}

Antes de comenzar el programa de desarrollo profesional se aplicó una encuesta de caracterización donde se recogió información general sobre la experiencia y formación de los formadores, incluido tipo de contrato (ver tabla 1). Además, se les preguntó por las 
distintas funciones que ellos desempeñaban en sus universidades, incluyendo enseñanza y apoyo de estudiantes en práctica (tutorías y supervisión).

Tabla 1. Muestra de formadores por universidades

\begin{tabular}{|l|c|c|c|}
\hline \multicolumn{1}{|c|}{ Tamaño (1) } & $\begin{array}{c}\text { Contrato Honorario } \\
\text { por horas }\end{array}$ & $\begin{array}{c}\text { Contrato Planta más } \\
\text { de media jornada }\end{array}$ & Total \\
\hline Grande o mediana & 5 & 4 & $11^{\mathrm{a}}$ \\
\hline Pequeña & 4 & 8 & $14^{\mathrm{a}}$ \\
\hline
\end{tabular}

Note: Grande o mediana se refiere a universidades de más de 100 estudiantes y Pequeña a universidades de menos de 100 estudiantes.

${ }^{a}$ Dos Formadores no reportan dedicación horaria.

Los tres focus groups fueron realizados justo antes de comenzar el programa de desarrollo profesional. Se organizaron tres grupos dirigidos por una psicóloga investigadora y una asistente de investigación. Los grupos fueron organizaron de manera de permitir cierta homogeneidad sobre los roles desempeñados (según lo reportado en la encuesta) y sobre los contextos de trabajo, pero a la vez permitiendo cierta heterogeneidad de manera de permitir discusión. En el grupo 1 la mayoría de los formadores estaban trabajando como tutores de práctica en universidades grandes o medianas. Los formadores de los grupos 2 y 3 eran principalmente profesores de cátedra en universidades con baja selectividad. Las universidades del grupo 2 eran principalmente universidades pequeñas en ciudades pequeñas y en el grupo 3 eran principalmente universidades pequeñas o medianas en grandes ciudades. La conversación fue grabada en video y audio. Cada focus group duró aproximadamente una hora y los formadores fueron invitados a conversar sobre dos tópicos principales: roles y actividades que ellos desarrollaban en sus universidades, y recursos disponibles para ayudarlos en su labor.

Dos meses después de los grupos focales se realizaron seis entrevistas, a tres formadores de mucha experiencia y a tres formadores con menor experiencia en formación inicial docente, representando universidades de distintas ciudades del país. Las entrevistas fueron organizadas para explorar en mayor profundidad temáticas discutidas en los grupos focales y de manera de contrastar más en detalle categorías de comparación sugeridas en los grupos. Por ejemplo, en los grupos focales se dedicó un tiempo considerable a discutir el rol de los formadores en el trabajo con estudiantes en práctica en universidades medianas y grandes de alta selectividad, pero no quedó muy claro cómo este trabajo se organizaba en universidades más pequeñas, por lo que se entrevistó a supervisores de práctica de dos universidades pequeñas. Debido a la relevancia que también adquirió la realización de roles diversos en los grupos focales, se entrevistó a un formador que mencionó realizar mucho trabajo de gestión y coordinación institucional y a dos formadores que mencionaron en el focus roles relacionados con la investigación en dos contextos diferentes (una universidad grande en ciudad grande y una muy pequeña en una universidad muy pequeña). Por último, se entrevistó a una formadora con alta experiencia en formación de profesores en general, pero no de matemáticas en particular, para explorar aspectos del contexto que pudiesen haber facilitado o dificultado este tránsito. 
Estudios Pedagógicos XLIV, $\mathrm{N}^{\circ}$ 3: 101-116, 2018

ENSEÑANDO MATEMÁTICAS A FUTUROS PROFESORES EN UN SISTEMA COMPLEJO: FORMADORES DE PROFESORES EN UNIVERSIDADES DE CHILE

\section{ANÁLISIS DE DATOS}

Tanto grupos focales como entrevistas fueron transcritas y se realizaron análisis temáticos (Braun \& Clarke, 2006). Inicialmente se aplicaron códigos altamente cercanos al dato para describir lo que se estaba diciendo, para luego avanzar a categorías más abstractas. Las categorías generales fueron incluyendo descripción de tareas, recursos y características de los contextos y conflictos o tensiones evidentes en el discurso. En etapas avanzadas de la codificación de tareas, se codificaron los roles asociados de acuerdo a los descritos por Lunenberg y colegas (2014) (ver tabla 2 más adelante). Luego de que cada intervención fue codificada, la información fue nuevamente analizada, contrastando descripciones de formadores que trabajaban en diferentes contextos. En este proceso, la comparación constante de casos y un análisis de cómo los recursos y demandas de las instituciones eran percibidas y reportadas por los formadores, fue central. El análisis fue realizado por tres de las cinco autoras en un archivo compartido en línea y numerosas reuniones de discusión de los análisis fueron llevadas a cabo en el proceso.

\section{RESULTADOS}

Los formadores de profesores describen su trabajo como diverso, sin embargo, cuando se analizaron en detalle las descripciones de las tareas que ellos realizan, se encontró que estos señalan mayoritariamente tareas que se relacionan con el cumplimiento de roles de enseñanza y supervisión de prácticas en establecimientos educacionales. Las descripciones de sus tareas incluyen planificación, evaluación, realización de clases, supervisión y tutoría de estudiantes en práctica. Esto último involucra facilitar su desempeño en tareas habituales (e.g. planificación) y coordinar el trabajo con los colegios. Algunos de ellos también mencionan la supervisión de tesis de estudiantes de pregrado y tener una cohorte de estudiantes a cargo (actuar como profesor jefe). Pocos mencionan tareas relacionadas con el desarrollo de curriculum e investigación (ver tabla 2).

El tema más relevante que emerge en el análisis es la relación que existe entre tipos de contratos en instituciones de distinto tamaño, roles ejercidos y políticas de reforma, y su influencia en cómo los formadores de profesores viven su trabajo y las identidades correspondientes.

Las instituciones medianas y grandes han desarrollado políticas de contratación para satisfacer necesidades específicas (profesores contratados para un rol específico como enseñar o supervisar). Las diferencias entre los formadores con contrato de tiempo completo versus profesores contratados por horas resultan en un sistema segregado con roles diferenciados al interior de las instituciones. Al hacer el análisis de los tipos de tareas reportadas y roles asociados en las universidades grandes, los profesores con jornadas completas o de alta dedicación horaria tienden a reportar mayor cantidad de tareas y roles asociados que sus colegas con jornadas de baja dedicación horaria y contratos a honorarios. Los profesores con contratos de alta dedicación reportan cumplir diversos roles dentro de la institución más allá de un foco centrado en la enseñanza y en la supervisión de prácticas (ej., gestión de equipos, investigación, desarrollo curricular). Por ejemplo, un profesor de planta mencionó que parte de su trabajo ha sido ir adquiriendo nuevos roles (y correspondientes nuevas identidades), lo que ha conllevado que su identidad como formador de profesores 
Tabla 2. Número de formadores que reportan los distintos roles en grupos focales y en encuestas.

\begin{tabular}{|l|l|c|c|c|}
\hline \multicolumn{2}{|c|}{} & \multicolumn{3}{|c|}{ Roles reportados en la encuesta } \\
\cline { 3 - 5 } \multicolumn{2}{|c|}{} & $\begin{array}{c}\text { Only } \\
\text { teaching }\end{array}$ & $\begin{array}{c}\text { Only } \\
\text { coaching }\end{array}$ & $\begin{array}{c}\text { Teaching - } \\
\text { Coaching }\end{array}$ \\
\hline \multirow{4}{*}{$\begin{array}{l}\text { Roles según el análisis } \\
\text { de grupos focales }\end{array}$} & Enseñanza & 12 & & 7 \\
\cline { 2 - 5 } & Supervisión de prácticas & & 6 & 7 \\
\cline { 2 - 5 } & Relación con escuelas & & & 2 \\
\cline { 2 - 5 } & Desarrollo curricular & 5 & & 1 \\
\cline { 2 - 5 } & Investigador & 4 & 1 & 1 \\
\cline { 2 - 5 } & Selección & 1 & & 1 \\
\hline
\end{tabular}

Note: a Enseñanza: Enseñar a estudiantes en salas universitarias (cátedras). Supervisión de prácticas: Guiar, tutorías a estudiantes en práctica. Relación con escuelas: Gestionar relaciones con escuelas para la realización de prácticas. Desarrollo curricular: Trabajo en desarrollo de curricular y planificación de programas. Investigación: Implementación, aplicación a fondos y publicación. Selección: Responsabilidad en admisión de estudiantes a los programas.

sea desplazada desde de la enseñanza y la supervisión de estudiantes hacia la dirección (supervisar y desarrollar pautas para que otros formadores de profesores apliquen):

Claro y eso implica que existen ciertos lineamientos o políticas institucionales que yo como directivo, como director de escuela, director de departamento, tengo que hacer solo 4 horas de docencia. El cambio directivo obliga a dedicar tiempo a la gestión y eso te descarga en docencia (...) Nuestro fuerte en docencia está principalmente con docentes hora, docente a honorarios. Por lo tanto, el equipo de docentes planta somos los encargados de poder y dar los lineamientos para que la docencia que hagan estos profesores, adjunto a profesores hora, sea consistentes con los lineamientos de la universidad (Entrevista formador cátedra, contrato jornada completa, Universidad grande).

La cita muestra cómo ciertas identidades (ser el director) son institucionalmente proveídas de estatus y poder en desmedro de otras tareas asociadas a identidades institucionalmente desprovistas de valor (la docencia). Los profesores (especialmente los profesores hora) son posicionados como receptores de lineamientos que son entregados desde la institución (la universidad) y de las personas en cargos de poder (quienes gestionan lo que se enseña).

En contraste, mayor cantidad de los profesores contratados por hora reportan realizar gran cantidad de tareas, pero usualmente asociadas sólo a la enseñanza o a la supervisión de alumnos en práctica. En varios casos, la manera como ellos describen las tareas que realizan evidencian como su trabajo se traduce en ser receptores de lo que perciben como pautas cerradas para la realización de su trabajo (algunos de ellos incluso reciben los PowerPoint para cada clase), las que a veces se encuentran en tensión con algunos de los requerimientos de su trabajo. Por ejemplo, algunos supervisores de estudiantes en práctica, contratados 
Estudios Pedagógicos XLIV, $\mathrm{N}^{\circ}$ 3: 101-116, 2018

ENSEÑANDO MATEMÁTICAS A FUTUROS PROFESORES EN UN SISTEMA COMPLEJO: FORMADORES DE PROFESORES EN UNIVERSIDADES DE CHILE

para desempeñar este rol, percibieron que el nuevo plan de estudios era demasiado exigente y estaba en tensión con las demandas de los estudiantes. Los estudiantes demandan más atención de sus necesidades en los colegios de práctica, poniendo en conflicto dos identidades: la del docente que enseña y la del que contenedor o soporte de las alumnas:

Nuestro rol como formador de profesores es abordar un conjunto numeroso de prácticas (...) enseñamos varias de esas prácticas, pero nos damos cuenta que en realidad nosotros necesitamos mucho más tiempo para que eso se aprenda bien. Se nos hace bastante corto el semestre para todo lo que tenemos que enseñar y tenemos clases sumamente recargadas (...) Además somos contenedores de las estudiantes... Y ahí también uno se tensiona un poco (...) Es esa contradicción desde el rol que uno tiene, como no puedo porque si se me dedico a apoyar y contener y a conversar más tiempo sobre la casuística, el estudiante va a dejar de aprender una práctica generativa que después se la voy a valorar en un trabajo (Grupo focal 1, formadora supervisora de práctica, contrato por hora, universidad grande).

Pese a que formadores contratados por hora en universidades de gran tamaño valoran los lineamientos y pautas entregadas por la institución (ej. son percibidas como profesionalizantes) perciben una subvaloración de su rol en conflicto con, por ejemplo, la identidad del formador como investigador.

Ahora tiene mucho que ver también con el tema de la remuneración lo que tu hagas. Tu categorización académica va a depender de si has publicado (...) y la verdad es que muchas veces... te pagan menos y trabajas más. Generalmente es así. O sea, si tienes un grupo más grande te van a pagar lo mismo que si... hay prácticas que se da un poco esta cosa irregular. Y, en la práctica de especialidad en matemática por lo menos, a mí se me paga una hora por alumno, pero también la demanda es muy alta ¿ya? Pero para poder hacer ascender en categorías académicas, tú tienes que ser investigador (Grupo focal 1 , formadora supervisora de práctica, contrato por hora, universidad mediana).

En comparación con las instituciones grandes y medianas, los formadores de profesores contratados por hora en las universidades pequeñas hacen descripciones que dan cuenta de menor acceso a recursos y apoyos de las instituciones. Por ejemplo, no reciben pautas claras sobre cómo implementar el plan de estudios, lo que es percibido como falta de recursos de inducción y formalización de la labor.

me contrataron, o sea me dijeron "Supervisor" y me hicieron una inducción muy rápida porque era yo el único que estaba ahí, los demás ya sabían y después me pasaron una guía que ellos tienen, como una pauta cómo tú vas supervisando. O sea, pero no me dijeron "mira tienes que tener esto". Por supuesto, "léete la pauta". La leí, pero hay cosas que todavía...quedan muchas dudas y eso... (Entrevista formador supervisor de práctica, contrato por hora, universidad pequeña).

Además, la mayoría de los profesores contratados por hora en universidades pequeñas experimentan condiciones de trabajo precarias. Se les paga dependiendo de los estudiantes que supervisan - horas que enseñan, no pasan tiempo en la universidad (no tienen oficina en 
la institución) y no tienen obligaciones contractuales. Uno de ellos habla sobre la falta de compromiso de sus estudiantes con el curso que dicta, sin enlazar esta falta de compromiso con la falta de apoyo de la institución:

los principales desafíos es tratar de que las alumnas entiendan de una o de otra forma que la supervisión es súper importante, o sea la práctica es muy importante para el desarrollo de su carrera profesional ¿ya? Porque ellas lo toman como... porque no es como una clase, llamémosla así, metódica, de que tienen que ir a clase dos veces por semana específicamente. Ellas la toman muy así, como... como cualquier cosa (Entrevista formador supervisor de práctica, contrato por hora, universidad pequeña).

Respecto de los formadores de universidades pequeñas con contratos de alta demanda horaria, perciben diferentes tensiones aquellos que han sido recientemente contratados para cumplir roles de investigación y docencia (formadores de poca experiencia en formación inicial), y aquellos que llevan largos contratos en la universidad y perciben sus identidades como formadores han cambiado producto de reformas curriculares (formadores con alta experiencia). Tres formadoras que participaron del estudio fueron recientemente contratadas en instituciones pequeñas debido a la reciente obtención de sus grados de doctor/a, o magíster en didáctica. Ellas mencionaron que en las instituciones les han otorgado recursos específicos para que desarrollen investigación (asignación de horas y fondos para asistentes de investigación), y recursos para implementar cambios en el plan de estudios (recursos para comprar material pedagógico).

Y hace dos años trabajo en la Universidad X [Universidad pequeña en ciudad pequeña] y la verdad es que yo llegué ahí, así como rompiendo el esquema (...) yo llegué con mi didáctica "lancémonos con el proyecto de comprar material" (...) y compraron el material y yo después con mi material y no que para arriba y para abajo, y empezamos a ver los programas también. Qué les faltaba, qué les sobraba, cómo estaban enfocados (Grupo focal 3, formadora docente, contrato tiempo completo, universidad pequeña).

En los tres casos, la entrega de recursos es ligada por las formadoras a una extensión de su rol a nuevos ámbitos. En el caso de la cita se observa una extensión de identidades relacionadas con la docencia a identidades relacionadas con el desarrollo curricular, mencionándose también la extensión de roles de supervisión y de relación con las escuelas con roles de investigación. Estas extensiones de los roles son narradas de forma en que las formadoras parecen tomar identidades de promotoras de cambios institucionales, en donde reformas y cambios son percibidos y narrados como proyectos personales y que motivan a convertirse en modelos a seguir para sus pares:

y yo dije "ya hay que trabajar con los estándares de egreso" y empecé el segundo semestre de a poquito ahí y claro, este año ya yo no soy bicho raro, estamos toda la universidad ya trabajando en el tema de cambiar el enfoque de los programas ¿cierto? De la enseñanza, desde una enseñanza por objetivos, a una enseñanza por competencia y ahí ahora todo el mundo se involucró en los estándares y analizamos los estándares de matemáticas y dice "ah es que el cambio que tú hiciste no se ha agarrado" y "ahora todo el mundo a cambiar" (Grupo focal 3, formadora docente, contrato tiempo completo, universidad pequeña). 
Mientras que los profesores novatos se posicionan como impulsores de cambio y manifiestan identidades de este tipo (ej. transitar desde ser la que rompe esquema a ya no ser el bicho raro o ser quien generó un cambio), los profesores más antiguos sienten que el contexto cambiante (principalmente por reformas curriculares en planes de enseñanza de las matemáticas, que por ejemplo se traducen en aumentos de cursos y cambios en enfoques de enseñanza) ha presionado para que sus identidades como profesores vayan modificándose también. En algunos casos, describen extensiones de roles en tensión con sus habilidades, capacidades o formación, como, por ejemplo, enseñar matemáticas aún cuando no son especialistas en el área, investigar y publicar aún cuando no poseen las calificaciones necesarias y cumplir roles de supervisión de sus pares sin autoridad. Muchas veces esta tensión es experimentada como dificultad:

... yo con una mención de hace 20 o más años, me pidieron hacerme cargo de matemática (...) para mí esto es como un ehm... nuevo. O sea, porque yo no soy matemática y yo la matemática que aprendí era del tipo algoritmo y qué sé yo, entonces yo voy a tener que dar cuenta, estoy dando vuelta mi cabeza, pero yo creo que voy a sufrir mucho también realmente, porque todo es distinto, es la forma distinta (Focus group 2, formadora docente, contrato tiempo completo, universidad pequeña).

Como se observa en la cita y en otras donde conflictos similares son expresados, las reformas contextuales y las presiones del medio son en algunos casos experimentadas como contradicciones de identidades, de quien soy (por ejemplo, formador general o profesor de profesores) versus en quién me debo convertir y que aún no soy (por ejemplo, matemático o investigador). Sin embargo, otros formadores experimentan los cambios y reformas curriculares como cambios en el tipo de profesor que deben ser (de su identidad de profesor) que no es experimentado en conflicto con lo que se es, sino como una oportunidad de desarrollo. Una formadora que narra este tipo de transición de identidades menciona cómo el cambio ha sido paulatino y ha estado acompañado por reconocimiento, valoración y libertad otorgada en la universidad en la que trabaja.

Es que yo creo que el cambio se ha ido produciendo en mí, en forma muy paulatina (...) he tenido que, y cuando te decía que he tenido un cambio en mi relación con los alumnos, también tiene que ver con centrar el proceso en el estudiante, en el que aprende. Y la verdad es que en la universidad yo me siento bastante reconocida en términos de "haga lo que quiera". O sea, si usted quiere armar un cuento, ármelo. porque la gente tiene confianza en lo que voy a hacer. Y eso ha sido para mí una cuestión muy grata y muy... me ha dado mucho espacio para hacer cosas (Entrevista formadora docente, contrato tiempo completo, universidad pequeña).

\section{CONCLUSIÓN Y DISCUSIÓN}

La complejidad y multiplicidad de roles e identidades que los formadores de profesores tienen que encarnar en instituciones de formación inicial docente están claramente influenciados por los contextos donde estas identidades deben ser representadas, los recursos disponibles y las políticas en desarrollo. Explorar la diversidad de experiencias 
de los formadores de profesores en estos contextos puede ofrecer importante información sobre cómo los cambios deben ser implementados y particularmente cómo se puede implicar a los formadores en movilizar estos cambios. Siguiendo lo anterior, el estudio de los diversos contextos en los que se forma a profesores puede ofrecer indicaciones acerca de cómo la formación inicial docente puede mejorar.

En el caso de Chile, un dinámico contexto de política educacional e importantes cambios curriculares (ver Cox et al., 2010) han impactado las políticas de contratación de formadores y los roles e identidades que ellos experimentan en el ejercicio de sus labores, aumentando la demanda de recursos que puedan apoyar la implementación de estos cambios. Este estudio mostró que los formadores experimentan como recursos de apoyo tanto lineamientos específicos sobre los requerimientos de la labor (principalmente formadores con contratos hora en roles específicos en universidades grandes), estatus otorgado por la extensión de roles y la entrega de libertad (formadores con experiencia en universidades pequeñas y grandes), y entrega de recursos concretos para promoción de roles relacionados con la investigación y el desarrollo curricular (nuevas contrataciones en universidades pequeñas). Los principales conflictos fueron identificados en formadores que narran historias en que los roles que se les asignan (y correspondientes identidades) están en contraposición con las identidades que ellos manifiestan tener (transitar a supervisor desde la enseñanza escolar, transitar a formador de profesores de matemáticas desde formador general, transitar a investigador desde docente de profesores). Estas dificultades fueron además mayoritariamente reconocidas en universidades pequeñas, usualmente ubicadas en ciudades más pequeñas, donde las presiones del medio externo se encuentran con una realidad de menores recursos (por la menor matrícula) y menor acceso a redes (menos pares en el interior de la universidad y menos contacto con otros formadores de otros contextos).

Los resultados de este estudio refuerzan varias conclusiones levantadas en investigaciones anteriores respecto del desarrollo de identidades profesionales y respecto de experiencias de inducción y desarrollo profesional específico de formadores de profesores. Respecto de la investigación en identidades profesionales este estudio refuerza la importancia de considerar aspectos contextuales locales (Akkerman \& Meijer, 2011; Beijaard et al., 2004) e influencias del contexto macro, incluyendo políticas y reformas educativas (Gellert et al., 2013; Simmons \& Thompson, 2007). Es importante relevar cómo las políticas educacionales y reformas generan cambios en las políticas generales y prácticas específicas de contratación institucionales que impactan directamente en la experiencia de los formadores. Considerar la relación entre estos tres niveles parece entonces fundamental (nivel personal - local/institucional - macro/político/social) (ver también Gonzalez-Vallejos, 2018).

Respecto de formadores de profesores y su experiencia individual, este estudio muestra evidencia de la existencia de múltiples tensiones en la labor y en sus correspondientes identidades profesionales en el contexto chileno, al igual que en otros contextos (cf. Loughran, 2011; Swennen et al., 2009). En la implementación de políticas de cambio y mejoramiento institucional en formación docente, este estudio releva la importancia de considerar identidades individuales y como estas se relacionan con las identidades requeridas por los nuevos contextos impulsados por las reformas. Los recursos necesarios para apoyar y promover cambios deberán estar en relación con potenciales tensiones. Por ejemplo, la generación de apoyo y soporte a las nuevas labores tanto de formadores con experiencia como de formadores novatos, principalmente orientados a la libertad 
Estudios Pedagógicos XLIV, $\mathrm{N}^{\circ}$ 3: 101-116, 2018

ENSEÑANDO MATEMÁTICAS A FUTUROS PROFESORES EN UN SISTEMA COMPLEJO: FORMADORES DE PROFESORES EN UNIVERSIDADES DE CHILE

con apoyo (por ejemplo, reconocimiento, recursos, etc.), parece promover compromisos personales con el cambio. En contraste, ampliación de roles sin apoyo, en transiciones que son experimentadas como conflictivas, parecen dificultar movilización para el cambio. Siguiendo esto es posible hipotetizar que encarnar múltiples identidades (la extensión de enseñanza y supervisión a investigación y desarrollo curricular) puede sentirse y experimentarse como un recurso y no necesariamente un estrés, como ha sido usualmente sugerido en la literatura (Lunenberg et al., 2014).

\section{REFERENCIAS BIBLIOGRÁFICAS}

Akkerman, S. F. \& Meijer, P. C. (2011). A dialogical approach to conceptualizing teacher identity. Teaching and teacher education, 27(2), 308-319.

Ávalos, B. (2002). Profesores para Chile: historia de un proyecto. Santiago: Ministerio de Educación.

Ávalos, B. (2014). La formación inicial docente en Chile: Tensiones entre políticas de apoyo y control. Estudios pedagógicos, 40(ESPECIAL), pág.11-28.

Ávalos, B. \& de Los Rios, D. (2013). Reform environment and teacher identity in Chile. En Diane B. Napier \& Suzanne Majhanovich. (eds) Education, Dominance and Identity. (pp. 153-175). Rotterdam: Sense Publishers.

Ávalos, B., \& Matus, C. (2010). La formación inicial docente en Chile desde una óptica internacional. Informe nacional del Estudio Internacional IEA TEDS-M. Santiago de Chile: Ministerio de Educación.

Battey, D. \& Franke, M. L. (2008) Transforming identities: Understanding teachers across professional development and classroom practice, Teacher Education Quarterly, 35(3), 127-149.

Beijaard, D., Meijer, P. C. \& Verloop, N. (2004). Reconsidering research on teachers' professional identity. Teaching and teacher education, 20(2), 107-128.

Beauchamp, C. \& Thomas, L. (2009). Understanding teacher identity: An overview of issues in the literature and implications for teacher education. Cambridge journal of education, 39(2), 175189.

Braun, V. \& Clarke, V. (2006). Using thematic analysis in psychology. Qualitative research in psychology, 3(2), 77-101.

Brunner, J. J. (2005). Guiar el mercado: Informe sobre la educación superior en Chile. Disponible en: http://www.uai.cl/images/sitio/docentes/documentos/chilebrunner.pdf

Celik, S. (2011). Characteristics and competencies for teacher educators: Addressing the need for improved professional standards in turkey. Australian Journal of Teacher Education, 36(4), 7387.

Cisternas, T. (2011). La investigación sobre formación docente en Chile: Territorios explorados e inexplorados. Calidad en la educación, (35), 131-164.

Comisión sobre Formación Inicial Docente (2005) Informe Comisión sobre Formación Inicial Docente. Santiago: Serie Bicentenario

Contreras, C. (2014). El desarrollo docente del formador de profesores: una propuesta orientada hacia el análisis de incidentes críticos auténticos. Estudios pedagógicos (Valdivia), 40 (ESPECIAL), 49-69.

Contreras, C., Monereo, C., \& Badia, A. (2010). Explorando en la identidad: ¿Cómo enfrentan los docentes universitarios los incidentes críticos que ocurren en las aulas de formación de futuros profesores? Estudios Pedagógicos, 36(2), 63-81.

Cox, C., Meckes, L. \& Bascopé, M. (2010). La institucionalidad formadora de profesores en Chile en la década del 2000: velocidad del mercado y parsimonia de las políticas. Pensamiento Educativo, 46(47), 205-45. 
Dengerink, J., Lunenberg, M. \& Kools, Q. (2015). What and how teacher educators prefer to learn. Journal of education for teaching, 41(1), 78-96.

Dinkelman, T., Margolis, J. \& Sikkenga, K. (2006). From teacher to teacher educator: Experiences, expectations, and expatriation. Studying Teacher Education, 2(1), 5-23.

Gellert, U., Espinoza, L \& Barbé, J. (2013) 'Being a mathematics teacher in times of reform', ZDM, 45(4), 535-545.

González-Vallejos, M. P. (2018). El estudio del formador latinoamericano: un campo de investigación 'en construcción'. Revista Internacional de Investigación en Educación, 10 (21), 35-54

Gresalfi, M. \& Cobb, P. (2011). Negotiating identities for mathematics teaching in the context of professional development, Journal for Research in Mathematics Education, 42(3), 270-304.

Guilfoyle, K. (1995). Constructing the meaning of teacher educator: The struggle to learn the roles. Teacher Education Quarterly, 11-26.

Holland, D., Lachicotte, W. J., Skinner, D. \& Cain, C. (1998). Identity and agency in cultural worlds. Cambridge, Massachusetts: Harvard University Press.

Koster, B., Brekelmans, M., Korthagen, F., \& Wubbels, T. (2005). Quality requirements for teacher educators. Teaching and teacher education, 21(2), 157-176.

Legard Larson, M. \& Kalmbach Phillips, D. (2005). Becoming a teacher of literacy: The struggle between authoritative discourses. Teaching Education, 16(4), 311-323.

Loughran, J. (2011). On Becoming a Teacher Educator. Journal of Education for Teaching, 37 (3), 279-291.

Lunenberg, M., Dengerink, J. \& Korthagen, F. (2014). The Profesional Teacher Educator: Roles, bahaviours and Professional Development of Teacher Educators. Rotterdam, Boston, Taipei: Sense Publishers.

Lunenberg, M., Korthagen, F. \& Swennen, A. (2007). The teacher educator as a role model. Teaching and teacher education, 23(5), 586-601.

Mecesup (2016). Financiamiento Institucional. Obtenido en: http://www.mecesup.cl/index2.php?id_ contenido $=15048 \& i d \_$portal $=59 \& i d \_$seccion $=3606$

Mineduc (2005). Informe Comisión sobre Formación Inicial Docente. Serie Bicentenario. Santiago.

Mineduc (2016). Identificación de elementos críticos para fortalecer la formación de profesores en el área de matemática de Pedagogía en Educación Básica en Chile.

Montenegro, H. \& Medina, L. (2014). Polifonía discursiva y procesos de cambio en los formadores de docentes: ¿Qué voces movilizan sus prácticas de enseñanza? Estudios pedagógicos (Valdivia), 40(ESPECIAL), 161-182.

Murray, J. \& Kosnik, C. (2011). Academic work and identities in teacher education. Journal of education for teaching, 37(3), 243-246.

Murray, J. \& Male, T. (2005). Becoming a teacher educator: Evidence from the field. Teaching and teacher education, 21(2), 125-142.

OECD (Organisation for Economic Co-operation and Development) (2005). Teachers matter. Attracting, developing and retraining effective teachers. Paris: OECD Publishing.

OECD (Organisation for Economic Co-operation and Development) (2011). Building a High-Quality Teaching Profession. Lessons from around the World. Paris: OECD Publishing.

Olsen, B. (2008). How reasons for entry into the profession illuminate teacher identity development. Teacher education quarterly, 35(3), 23-40.

Putnam, R. T. \& Borko, H. (2000). What do new views of knowledge and thinking have to say about research on teacher learning? Educational researcher, 29(1), 4-15.

Sachs, J. (2005). Teacher education and the development of professional identity: Learning to be a teacher. En P. Denicolo y M. Kompf (Eds.), Connecting policy and practice: Challenges for teaching and learning in schools and universities (pp. 5-21). Oxford: Routledge.

Shagrir, L. (2015). Working with students in higher education-professional conceptions of teacher educators. Teaching in higher education, 20(8), 783-794. 
Estudios Pedagógicos XLIV, $\mathrm{N}^{\circ}$ 3: 101-116, 2018

ENSEÑANDO MATEMÁTICAS A FUTUROS PROFESORES EN UN SISTEMA COMPLEJO: FORMADORES DE

PROFESORES EN UNIVERSIDADES DE CHILE

Simmons, R. \& Thompson, R. (2007). Teacher educators in post-compulsory education: gender, discourse and power. Journal of vocational education and training, 59(4), 517-533.

Smagorinsky, P., Cook, L. S., Moore, C., Jackson, A. Y. \& Fry, P. G. (2004). Tensions in learning to teach: Accommodation and the development of a teaching identity. Journal of teacher education, $55(1), 8-24$.

Smith, K. (2005). Teacher educators' expertise: What do novice teachers and teacher educators say? Teaching and teacher education, 21(2), 177-192.

Sotomayor, C. \& Gysling, J. (2011). Estándares y regulación de calidad de la formación de profesores: discusión del caso chileno desde una perspectiva comparada. Calidad en la educación, (35), 91129.

Swennen, A., Shagrir, L. \& Cooper, M. (2009). Becoming a teacher educator: Voices of beginning teacher educators. En Anja Swennen \& Marcel van der Klink (eds) Becoming a teacher educator (pp. 91-102). Springer Netherlands.

Varas, L., Felmer, P., Gálvez, G., Lewin, R., Martínez, C., Navarro, S., Ortiz, A. \& Schwarze, G. (2008). Oportunidades de preparación para enseñar matemáticas de futuros profesores de educación general básica en Chile. Calidad en la Educación, 29, 64-88.

Williams, J. \& Berry, A. (2016). Boundary Crossing and the Professional Learning of Teacher Educators in New International Contexts. Studying Teacher Education, 12(2), 135-151.

Williams, J. \& Ritter, J. K. (2010). Constructing new professional identities through self-study: From teacher to teacher educator. Professional development in education, 36(1-2), 77-92.

Zeichner, K. (2005). Becoming a teacher educator: A personal perspective. Teaching and teacher education, 21(2), 117-124. 